\title{
Krise der Geburtshilfe in den USA
}

Wenn bei einer Geburt das Baby nicht vollkommen gesund ist oder bei der Mutter irgendwelche Komplikationen auftreten, werden heutzutage in den USA die Geburtshelfer vor Gericht gezogen. Und das unabhängig davon, ob ein Kunstfehler vorliegt oder ob die Frau ohne Schwangerschaftsversorgung mit einer Komplikation bei der Notfallaufnahme ankommt und vom Dienstarzt, der die Frau zum ersten Mal sieht, entbunden wird.

Sogenannte «Trail Lawyers» bieten ihre Dienste auf Flugblättern und Plakatwänden an. Schwerbehinderte Kinder werden im Gerichtssaal den Geschworenen vorgeführt. Aus lauter Erbarmen und weil ja «die Versicherung sowieso alles bezahlt», sind Millionenurteile an der Tagesordnung, 8,1 Millionen kürzlich in Utah für einen «Bad Baby Case». Im Durchschnitt erhalten aber die Patienten nur 13 Cent pro Dollar Schadenersatz. Der Rest geht an die Anwälte, die auf Erfolgsbasis arbeiten, und an die Gerichtsexperten.

Diese «Klag jetzt, frag später»-(oder überhaupt nie-)Tendenz führte zu einer beispiellosen Krise im amerikanischen Gesundheitswesen, mit der sich jetzt sogar die Bush-Regierung abzugeben beginnt. Die Medizin ist nicht das einzige Gebiet, das unter einer Haftpflichtkrise leidet. Konservative Schätzungen der Kosten des USHaftpflichtsystems belaufen sich auf fast 200 Milliarden, etwa 1,8\% des BSP. Da aber die meisten US-Politiker Anwälte sind, fehlt vorläufig der politische Wille, die Gesetze zu ändern oder wenigstens eine Limite für «Pain and Suffering»Entschädigungen zu setzen.

Mehr als drei Viertel der US-Geburtshelfer sind mindestens einmal vor Gericht verklagt worden. Laut einer Umfrage des American College of Obstetricians and Gynecologists (ACOG) haben etwa 20\% der Gynäkologen die Geburtshilfe aus legal-politischen Gründen aufgegeben. Weitere $60 \%$ haben ihre Praxis auf «low risk»Fälle limitiert.

Einer der Hauptgründe, dass Gynäkologen die Geburtshilfe aufgeben, sind die Kosten ihrer «Malpractice»-Versicherungen im Vergleich zu dem, was ihnen von den privaten Versicherungen oder vom Staat (Medicaid) als Honorar bezahlt wird. Die Versicherungsprämien haben sich in den letzten paar Jahren mehr als verdoppelt und belaufen sich schon in vielen Staaten auf dreistellige Beträge, ohne dass die Honorare entsprechend anstiegen. In Utah kostet die Versicherung dieses Jahr \$ 86000; im Jahr 2000 waren es noch $\$ 41000$. Das heisst, dass bei einer Entschädigung von \$1200-1300 pro Geburt (inklusive 12-14 pränataler Visiten und 6 Wochen postnataler Versorgung) ungefähr die ersten 70 Geburten pro Arzt und Jahr an die Bezahlung der Versicherungsprämie gehen! Die übrigen Praxisunkosten, die sich in Utah auf 60-65\% des Umsatzes belaufen, kommen dann noch dazu. Medicaid bezahlt in Utah sogar nur etwa \$1000 pro Geburt.

Die Gynäkologen sind unter immer grösser werdendem Produktionsdruck und können es sich zum Beispiel nicht mehr leisten, längere Ferien zu nehmen oder Teilzeit zu arbeiten. Nationale Studien zeigen, dass 30-50\% der Ärzte an Depressionen und Burn-out leiden. Viele pensionieren sich ein bis zwei Jahrzehnte früher als noch vor ein paar Jahren oder wechseln den Beruf. Einige studieren Jurisprudenz.

Kein Wunder, dass in Utah 70\% der Gynäkologen über 45 Jahre alt sind und bestehende Praxen Mühe haben, neue Partner zu finden. Neu in die Praxis eintretende Ärzte beginnen ihre Karriere durchschnittlich mit über $\$ 100000$ Ausbildungsschulden und ziehen die wenigen Staaten, die wegen lokaler Gesetzgebung tiefere Haftpflichtversicherungsprämien und bessere Bezahlung haben, vor.

Die Situation ist noch viel prekärer in den ländlichen Gegenden, wo die Allgemeinpraktiker, die dort bisher meist die Geburtshilfe leiteten, mehrheitlich die Geburtshilfe aufgeben mussten, weil für sie die Versicherungsprämien mit den wenigen Geburten, die sie pro Jahr leiten, nicht mehr erschwinglich waren oder weil das lokale Spital aus dem gleichen Grund die Geburtsabteilung schloss. Das Resultat ist, dass in vielen Gemeinden in Utah die nächste Geburtsabteilung mehr als 200 Kilometer entfernt liegt. Schwangere müssen vor der Geburt in die Nähe eines Spitals ziehen oder, wenn die Wehen beginnen, per Auto losrasen. Solange das Kind unterwegs auf die Welt kommt, wird dann niemand verklagt, auch wenn's schiefgeht. Wenn aber die blockierte Geburt im Spital endet, wird der Dienstarzt verklagt, da er ja sicher etwas falsch gemacht haben muss.

Aber auch in der Stadt wird es langsam schwierig, einen Termin mit einem Gynäkologen zu erhalten. Die Praxen der Gynäkologen 
sind praktisch alle für neue Patientinnen geschlossen. Viele Frauen finden keine oder nur späte pränatale Versorgung, sogar wenn sie eine private Versicherung haben; viele Praxen nehmen keine Medicaid-Patientinnen mehr an. Die Frühgeburtenrate und andere Indikatoren fehlender medizinischer Versorgung steigen.

Die Hebammen, die in den USA etwa 7,5\% der Geburten leiten, verloren letztes Jahr die von ihrem nationalen Berufsverband offerierte Haftpflichtversicherung. Vielen gelang es nicht, eine neue Versicherung $\mathrm{zu}$ finden, und sie mussten ihre Praxen schliessen.

Dafür haben wir jetzt in Utah eine Gesetzesvorlage, die Laienhebammen erlauben würde,
Hausgeburten zu leiten, Labor- und Ultraschalluntersuchungen $\mathrm{zu}$ verordnen und $\mathrm{zu}$ interpretieren und parenterale Medikamente zu verabreichen. Das Ganze hätte dann noch einen Paragraphen, der erklärt, dass diese Laienhebammen nicht Medizin praktizierten und daher von der Haftpflicht dispensiert seien.

Die Krise der Geburtshilfe in den USA ist beängstigend und sollte für die Schweiz und andere Länder eine Warnung sein.

Dr. med. Regula E. Bürki, ehemalige Präsidentin, Utah Section ACOG, Salt Lake City 